\title{
Early and Late Onset Sepsis in Late Preterm Infants
}

\author{
Michael Cohen-Wolkowiez, MD, Cassandra Moran, DO, Daniel K. Benjamin, PhD, C. Michael \\ Cotten, MD, MHS, Reese H. Clark, MD, Daniel K. Benjamin Jr., MD, MPH, PhD, and P. Brian \\ Smith, MD, MHS \\ Department of Pediatrics (Drs Cohen-Wolkowiez, Smith, Cotten, Moran and Benjamin Jr.), Duke \\ University, Durham, NC; Duke Clinical Research Institute (Drs Cohen-Wolkowiez, Smith, and \\ Benjamin Jr.), Durham, NC; Department of Economics (Dr Benjamin), Clemson University, \\ Clemson, SC; Pediatrix-Obstetrix Center for Research and Education (Dr Clark), Sunrise, FL
}

\section{Abstract}

Background-Preterm birth is increasing worldwide, and late preterm births, which comprise more than $70 \%$ of all preterm births, account for much of the increase. Early and late onset sepsis results in significant mortality in extremely preterm infants, but little is known about sepsis outcomes in late preterm infants.

Methods-This is an observational cohort study of infants < 121 days of age $(119,130$ infants less than or equal to 3 days of life and 106,142 infants between 4 and 120 days of life) with estimated gestational age at birth between 34 and 36 weeks, admitted to 248 neonatal intensive care units in the United States between 1996 and 2007.

Results-During the study period, the cumulative incidence of early and late onset sepsis was 4.42 and 6.30 episodes per 1000 admissions, respectively. Gram-positive organisms caused the majority of early and late onset sepsis episodes. Infants with early onset sepsis caused by Gram-negative rods and infants with late onset sepsis were more likely to die than their peers with sterile blood cultures (OR 4.39, 95\% CI 1.71-11.23, $P=0.002$; and OR 3.37, 95\% CI 2.35-4.84, $P<0.001$, respectively).

Conclusion-Late preterm infants demonstrate specific infection rates, pathogen distribution, and mortality associated with early and late onset sepsis. The results of this study are generalizable to late preterm infants admitted to the special care nursery or neonatal intensive care unit.

\section{Keywords}

blood culture; neonate; prematurity; infection; near term

\section{INTRODUCTION}

The incidence of preterm births ( $<37$ weeks gestation) is increasing in many countries around the world and has become a global health concern.(1-4) More than 70\% of preterm infants are born between 34 and 36 weeks gestation (late preterm).(1,2,4,5) Most large series describing the epidemiology of neonatal sepsis are limited to infants with very low gestational age $(<33$ weeks) or very low birth weights (VLBW, $<1500 \mathrm{~g}$ birth weight).(6-9)

Late preterm infants are traditionally perceived by clinicians as physiologically similar to fullterm infants and are often treated similarly. Recent evidence suggests, however, that late

Corresponding Author: Danny Benjamin, MD, PhD, MPH, Associate Professor, Duke University, Pediatrics, 2400 Pratt St., Duke Clinical Research Institute, Durham, NC 27715; p: 919-668-8295; f: 919-681-9457; danny.benjamin@ duke.edu.

The authors declare that they have no conflicts of interest to disclose. 
preterm infants (relative to full-term infants) are diagnosed with culture-proven sepsis more frequently, have increased sepsis-related mortality, and have a substantial increased risk for morbidity and mortality.(10-14) Studies describing the epidemiology of early onset sepsis (EOS) and late onset sepsis (LOS) in late preterm infants are lacking, therefore, the United States National Institute of Child Health and Human Development of the National Institutes of Health issued an agenda to increase knowledge and understanding of complications related to late preterm birth.(15) Towards this end, we aimed to evaluate risk factors, pathogen distribution, cumulative incidence, and mortality of EOS and LOS in a large cohort of late preterm infants admitted to 248 neonatal intensive care units (NICU) in the United States.

\section{MATERIALS AND METHODS}

\section{Study Design and Setting}

We examined prospectively collected data from a cohort of infants $<121$ days of age with estimated gestational age (EGA) at birth of 34 to 36 weeks, admitted to 248 NICUs managed by the Pediatrix Medical Group in the United States between 1996 and 2007. The database was created using a computer-assisted tool that generates clinical progress notes. The accuracy and quality of the database relies upon the neonatologist entering the data. We collected neonatal and maternal demographic data, and we gathered information on blood cultures drawn for each patient during the first 120 days of life. Patients with missing blood culture data were excluded from the analysis. The microbiological data extracted from the physician computerized notes was not verified with microbiological culture records.

\section{Definitions and Participants}

We defined late preterm infants as infants born with an EGA of 34-36 weeks, inclusive, according to the criteria developed by the National Institute of Child Health and Human Development Workshop on Optimizing Care and Outcome of Late Preterm Infants.(15) Gestational age was determined by the best estimate of last menstrual period, obstetrical record, and clinical examination. We defined EOS and LOS as a positive blood culture during the first 3 days of life and between 4 and 120 days of life, respectively. Multiple positive blood cultures for the same organism within 21 days were considered a single episode of sepsis. Only blood culture results from Pediatrix institutions were captured. Maternal antibiotic use was recorded by the neonatologist on admission of the infant to the NICU.

We defined a definite coagulase-negative Staphylococcus (CoNS) sepsis as 2 positive blood cultures for CoNS drawn on the same day;(16) probable CoNS sepsis as 2 positive blood cultures for CoNS within a 4-day period, 3 positive blood cultures for CoNS within a 7-day period, or 4 positive blood cultures for CONS within a 10-day period; and possible sepsis as a culture positive for CoNS that did not meet criteria for definite or probable CoNS sepsis. We included definite and probable CoNS sepsis in the analysis.

We excluded the following observations from the analysis: 1) infants who died in the first 24 hours of life and who did not have a blood culture drawn ( $n=130)$; 2 ) sepsis episodes for organisms considered contaminants including non-speciated streptococci $(\mathrm{n}=108)$, Bacillus sp. $(\mathrm{n}=25)$, Corynebacterium sp. $(\mathrm{n}=7)$, diphtheroids sp. $(\mathrm{n}=8)$, Gram-positive rods $(\mathrm{n}=$ 33, not including Listeria sp.), Lactobacillus sp. $(\mathrm{n}=1)$, Micrococcus $\mathrm{sp} .(\mathrm{n}=13)$, Stomatococcus sp. $(\mathrm{n}=2)$, and Bacteroides sp. $(\mathrm{n}=1)$; and 3$)$ infants missing discharge date $(n=7)$. We grouped pathogenic organisms into 3 clinically relevant categories: Gram-negative rods (GNR), Gram-positive cocci (GPC), and yeast. 


\section{Statistical Methods}

The unit of observation for this analysis was the infant. We treated EOS and LOS as dichotomous variables and assessed maternal and infant risk factors for EOS and LOS by multivariable logistic regression. We evaluated causative agents and cumulative incidence for EOS and LOS among late preterm infants; we assessed mortality by organism; and we examined the risk of death between different organism groups using 2-sample proportioncomparison test. We determined by logistic regression the likelihood of death-adjusted for gestational age, birth weight, and 5-minute Apgar score - between infants with sepsis and those with sterile blood cultures. We calculated the cumulative incidence of EOS and LOS by the number of new cases (counted once) divided by the total number of admissions at study baseline; we calculated the LOS infection rate by the number of new cases (counted once) divided by the total number of hospital days; we determined the number of infection episodes per 1000 NICU admissions (EOS) and 1000 hospital days (LOS) by organism; and we examined Escherichia coli and group-B streptococci (GBS) EOS episodes by year. We compared Escherichia coli and GBS annual infection episodes between 1997 and 2007. For infants with LOS, we examined the number of infection episodes by organism group and day of life-adjusted by the number of infants present in the NICU on a given day. STATA 10 (College Station, TX) was used to perform the statistical analysis. Significance for all tests was established at a $P$ value of $<0.05$. Permission to conduct this analysis was provided by the Duke University Institutional Review Board.

\section{RESULTS}

From 1996 through 2007, the Pediatrix Medical Group cared for 119,130 late preterm infants $\leq 3$ days of life and 106,142 late preterm infants 4 to 120 days of life in the NICUs. Infants between the ages of 0 and 3 days had a mean EGA at birth of 34.9 weeks (SD, \pm 0.81 ) and a mean birth weight of 2431 grams $( \pm 510)$. Late preterm infants present in the NICU after the third day of life had similar means for EGA at birth and birth weight (34.9 weeks [ \pm 0.81$]$ and 2403 grams [ \pm 500$]$, respectively).

\section{Early Onset Sepsis}

The majority $(69.0 \%, 82,263 / 119,130)$ of late preterm infants admitted to the NICU within the first 3 postnatal days had at least 1 blood culture obtained. Only $0.6 \%(527 / 82,263)$ of infants evaluated with a blood culture met our definition of EOS. A total of 531 episodes of EOS were documented; 4 infants had 2 pathogens identified. The EOS cumulative incidence over the study period was 4.42 episodes per $1000 \mathrm{NICU}$ admissions. Demographic data for patients with EOS are presented in Table 1 (online only).

Risk factors for EOS among late preterm infants who had a blood culture drawn included Hispanic ethnicity (OR 1.24; 95\% CI 1.01-1.53; $P=0.04$ ), birth weight between 2500 and 3499 grams (OR 1.37; 95\% CI 1.01-1.87; $P=0.04$ ), and Cesarean delivery (OR 1.04; 95\% CI $1.01-1.08 ; P=0.01)$. Late preterm infants whose mothers received prenatal antibiotics were less likely to develop EOS (OR 0.65; 95\% CI 0.53-0.80; $P<0.001$ ) (Figure 1, online only).

Gram-positive organisms caused most episodes of EOS (66.4\%, 353/531), Gram-negative organisms accounted for $27.3 \%$ (145/531) of EOS, and yeast for $0.8 \%$ (4/531). GBS, Escherichia coli, and Staphylococcus aureus accounted for most EOS episodes within this group of infants (Table 2). During the study period, the number of GBS EOS episodes among late preterm infants decreased from 3.29 to 0.74 per 1000 NICU admissions $(P=0.03)$ in 1997 and 2007, respectively (Figure 2). The incidence of EOS caused by Escherichia coli also decreased during this same time period ( 1.46 to 0.74 episodes per $1000 \mathrm{NICU}$ admissions, respectively; $P=0.39$ ). 
Mortality of all infants during the first 3 days of life was $0.7 \%(805 / 119,130)$. Of the 527 infants with EOS, $1.3 \%$ died (7/527). Mortality was higher in infants with Gram-negative sepsis $(19.1 \%, 4 / 21)$ compared with Gram-positive sepsis $(1.1 \%, 2 / 175)$. No deaths were associated with GBS or yeast (0/120 and 0/4, respectively). After adjusting for gestational age, birth weight, and 5-minute Apgar score, infants infected with GNR organisms were more likely to die than infants with negative blood cultures (OR 4.39; 95\% CI 1.71-11.23; $P=0.002$ ).

\section{Late Onset Sepsis}

In contrast with the EOS cohort, only $6.2 \%(6529 / 106,142)$ of late preterm infants between 4 and 120 days of life had at least 1 blood culture obtained. Overall, 10.2\% (669/6529) of infants evaluated with a blood culture had a total of 803 episodes of LOS; 107 had multiple episodes ( 85 had 2 episodes, 17 had 3 episodes, 3 had 4 episodes and 1 had 5 episodes). The LOS cumulative incidence was 6.30 episodes per 1000 NICU admissions and the LOS infection rate was 0.76 episodes per 1000 hospital days. See Table 1 for demographic data of infants with LOS.

The probability of LOS was higher in late preterm infants born to mothers aged 11-19 years compared with those born to mothers aged $20-29$ years (OR 1.34; 95\% CI 1.06-1.69; $P=$ $0.01)$. LOS was also more frequent in late preterm infants with 5-minute Apgar scores $0-3$ and 4-6 (OR 2.09; 95\% CI 1.15-2.19, and OR 1.59; 95\% CI 1.21-3.59, respectively; $P<0.007$ ) (Figure 1, online only).

The majority of LOS episodes were caused by Gram-positive organisms $(59.4 \%, 477 / 803)$, followed by Gram-negative organisms $(30.7 \%, 247 / 803)$ and yeast $(7.7 \%, 62 / 803)$. The most commonly observed pathogens responsible for LOS included CoNS, Staphylococcus aureus, and Escherichia coli (Table 2). Of 129 episodes of CoNS LOS, 32.5\% (42/129) were classified as definite and $67.4 \%(87 / 129)$ as probable. None $(0 / 25)$ of the patients with definite CoNS LOS died; $4.0 \%$ (2/50) of patients with probable CoNS LOS died. The incidence of LOS episodes increased with increasing postnatal days, and the pathogen distribution changed little during that period (Figure 3).

Overall, $0.4 \%(480 / 106,142)$ of late preterm infants between 4 to 120 days of life died. Of the 669 infants with LOS, 7.0\% (47/669) died. After adjusting for gestational age, 5-minute Apgar score, and birth weight, infants with LOS were more likely to die than infants without sepsis (OR 3.37; 95\% CI 2.35-4.84; $P<0.001$ ). Mortality varied by organism and was higher for episodes due to non-speciated GNR $(24.0 \%, 6 / 25)$; Pseudomonas sp. $(40.0 \%, 4 / 10)$; Acinetobacter sp.(12.5\%, 1/8); non-speciated GPC (8.3\%, 13/155); Enterococcus sp. (6.6\%, 4/60); and Candida sp. (6.4\%, 4/62).

\section{DISCUSSION}

In the present study, the majority (69.0\%) of late preterm infants admitted to the NICU were evaluated for sepsis during the first 3 days of life. It is unclear, however, if the sepsis evaluations were prompted by clinical signs of sepsis or by the premature birth. Only $0.4 \%(527 / 119,130)$ of infants had a confirmed episode of EOS. Infants born to mothers who received prenatal antibiotics were less likely to have a positive blood culture. However, it is impossible to determine if administration of prenatal antibiotics decreased the likelihood of EOS or if it reduced the probability of a positive blood culture. Similarly, in a recent single-center study conducted between 1988 and 2005, 0.3\% (67/21,771) of late preterm infants were diagnosed with culture-proven sepsis.(11) Currently, there are no management guidelines addressing the need for sepsis evaluations in late preterm infants. The high proportion of infants evaluated with a blood culture and the small number of infection episodes suggests that the yield from 
sepsis evaluations in the majority of late preterm infants is low. Among very premature infants sepsis evaluations and EOS rates are higher (90\% and 1-2\%, respectively). $(9,17)$

Previously identified risk factors for EOS among very premature infants include low gestational age and prolonged rupture of amniotic membranes.(17)In the present investigation, however, the frequency of EOS among late preterm infants with lower gestational age (34 weeks EGA) was not higher than that observed in more mature infants (36 weeks EGA) [0.4 vs. $0.5 \%, \mathrm{P}=0.126]$. In addition, infants of higher birth weights had a higher frequency of EOS. This could be related to selection bias of late preterm infants with higher birth weight and EGA who are admitted to the NICU because of clinical signs compatible with sepsis, rather than because of their prematurity or low birth weight. A third of the patients in this cohort were Hispanic, and these patients had a greater frequency of EOS (over 20\%) when compared with infants of other races. Factors such as delayed or limited access to healthcare and low rates of prenatal care in this population may account for this finding.(18) Similar trends have been observed with other minorities; African- American newborns have higher rates of GBS EOS when compared with white infants. $(19,20)$

Since the introduction of GBS intrapartum prophylaxis, several institutions in the United States have reported a shifted distribution of pathogenic agents responsible for neonatal sepsis towards a predominance of GNRs, specifically Escherichia coli. $(9,17)$ This was not observed in the present study. GBS was responsible for $22.6 \%$ of Gram-positive EOS episodes, and the annual number of episodes decreased during the study period. This is predictable based on the increasing proportion of mothers receiving prenatal antibiotics as part of the introduction by the Centers for Disease Control (CDC) of intra-partum GBS antibiotic prophylaxis in 1996 and their modification in 2002. Candida as a causative organism for EOS in late preterm infants was rare $(0.7 \%)$.

Estimates of late preterm mortality attributed to EOS vary greatly, ranging from 0.05-0.6 per 1000 live births.(10,13)Similarly, we observed 0.06 EOS deaths per 1000 NICU admissions and a mortality of $1.3 \%$ among late preterm infants with EOS. In contrast, documented mortality among very premature infants with EOS is much higher (over 30\%). $(9,21)$ Late preterm infants infected with GNRs in the present study had higher mortality when compared to those with sterile blood cultures or infants infected by GPC. Similar case fatality rates associated with GNR sepsis have been observed among other NICU populations.(22-24)

When compared with infants in the EOS group, only the minority (6.2\%) of late preterm infants were evaluated with a blood culture and $0.6 \%$ had LOS. Notably, of all late preterm infants in the present study who had at least 1 blood culture drawn, $10.2 \%$ had culture-proven LOS. It is likely that the majority of late preterm infants who remain in the NICU past 3 days of life are relatively healthy and therefore are not automatically evaluated for sepsis. If a sepsis evaluation is performed, however, the likelihood of finding a true episode of sepsis is high ( 1 in 10 patients evaluated) relative to late preterm infants evaluated for EOS ( 1 in 167 patients evaluated).

The LOS rate in the present study was similar to that observed among infants $\geq 33$ EGA evaluated by the Neonatal Research Network (LOS rate 0.78 per 1000 hospital days).(7) The pathogen distribution of LOS was also similar to that reported for other populations in the NICU in that most episodes of LOS were caused by Gram-positive organisms, and the highest LOS rates were attributed to CoNS. $(7,25,26)$ Staphylococcus aureus and Escherichia coli were the second and third most prevalent organisms in late preterm infants with LOS, respectively. Given the predominance of GPC - other than GBS - it can be speculated that most episodes of LOS arose from the presence of an intravascular device. Patients with LOS had a higher incidence of Gram-negative and Gram-positive sepsis when compared to patients with EOS. This was probably related to prolonged stay in the NICU and higher exposure to nosocomially 
acquired organisms. Even though strict criteria were used to define definite CoNS sepsis, a higher number of patients with probable CoNS sepsis died, $4.0 \%(2 / 50)$ vs. $0 \%(0 / 25)$. While no causal relationship can be established, this finding raises suspicion as to whether definite or probable CoNS sepsis in this study reflects true infections. Eight percent of LOS episodes were caused by Candida sp, which is higher than the proportion of EOS caused by Candida. This observation is consistent with previous reports demonstrating the peak of neonatal Candida infections between 11 to 20 postnatal days.(27)

Mortality was low among late preterm infants older than 3 days admitted to the NICU $(0.4 \%)$; however, $7.0 \%$ of infants with LOS died. Moreover, late preterm infants with LOS were more likely to die relative to their peers with sterile blood cultures (OR 3.37; 95\% CI 2.35-4.84). The highest mortality was observed among sepsis episodes caused by Gram-negative organisms. Specifically, 40.0\% (4/10) of LOS episodes caused by Pseudomonas aeruginosa resulted in death. Other investigators have reported similar mortality associated with Pseudomonas aeruginosa. $(28,29)$

The present study is the largest evaluation of sepsis in late preterm infants and the first that specifically addresses characteristics of EOS and LOS in this unique population. Strengths of this report include a large sample size; population diversity from academic and community institutions; quantification of every blood culture obtained for each patient during the hospitalization; inclusion of maternal and infant risk factors for sepsis; and a contemporary study period after the introduction of intrapartum GBS antibiotic prophylaxis. This study is limited by lack of documentation of clinical signs or laboratory findings supportive of sepsis; lack of information on presence and duration of central catheters and congenital anomalies requiring surgery; lack of criteria for admission to the NICU; no direct confirmation of data obtained from outborn infants; and no maternal antimicrobial timing and dosing information. The percentage of infants with EOS exposed to intrapartum antibiotics was only $29 \%$. We were limited by available data in our ability to assess whether these mothers had risk factors as defined by the CDC for early onset GBS infection. Our results are generalizable to those institutions where late premature infants are admitted to the special care nursery or NICU.

\section{Acknowledgments}

Dr. Benjamin received support from NICHD HD-044799-04 and NCRR 1UL 1RR024128-02; Dr. Smith received support from NCRR 1UL 1RR024128-02. All data were obtained from the Pediatrix Medical Group database; however, this study did not receive any financial sponsorship from Pediatrix.

\section{References}

1. Barros FC, Victora CG, Barros AJ, et al. The challenge of reducing neonatal mortality in middleincome countries: findings from three Brazilian birth cohorts in 1982, 1993, and 2004. Lancet 2005;365:847-854. [PubMed: 15752528]

2. Buitendijk S, Zeitlin J, Cuttini M, et al. Indicators of fetal and infant health outcomes. Eur J Obstet Gynecol Reprod Biol 2003;111(Suppl 1):S66-77. [PubMed: 14642321]

3. Martin JA, Hamilton BE, Sutton PD, et al. Births: final data for 2005. Natl Vital Stat Rep 2007;56:1103. [PubMed: 18277471]

4. Moser K, Macfarlane A, Chow YH, et al. Introducing new data on gestation-specific infant mortality among babies born in 2005 in England and Wales. Health Stat Q 2007:13-27. [PubMed: 17894197]

5. Davidoff MJ, Dias T, Damus K, et al. Changes in the gestational age distribution among U.S. singleton births: impact on rates of late preterm birth, 1992 to 2002. Semin Perinatol 2006;30:8-15. [PubMed: 16549207]

6. Doctor BA, Newman N, Minich NM, et al. Clinical outcomes of neonatal meningitis in very-low birthweight infants. Clin Pediatr (Phila) 2001;40:473-480. [PubMed: 11583045] 
7. Stoll BJ, Hansen N, Fanaroff AA, et al. Late-onset sepsis in very low birth weight neonates: the experience of the NICHD Neonatal Research Network. Pediatrics 2002;110:285-291. [PubMed: 12165580]

8. Stoll BJ, Hansen NI, Adams-Chapman I, et al. Neurodevelopmental and growth impairment among extremely low-birth-weight infants with neonatal infection. JAMA 2004;292:2357-2365. [PubMed: 15547163]

9. Stoll BJ, Hansen NI, Higgins RD, et al. Very low birth weight preterm infants with early onset neonatal sepsis: the predominance of gram-negative infections continues in the National Institute of Child Health and Human Development Neonatal Research Network, 2002-2003. Pediatr Infect Dis J 2005;24:635-639. [PubMed: 15999007]

10. Kramer MS, Demissie K, Yang H, et al. The contribution of mild and moderate preterm birth to infant mortality. Fetal and Infant Health Study Group of the Canadian Perinatal Surveillance System. JAMA 2000;284:843-849. [PubMed: 10938173]

11. McIntire DD, Leveno KJ. Neonatal mortality and morbidity rates in late preterm births compared with births at term. Obstet Gynecol 2008;111:35-41. [PubMed: 18165390]

12. Shapiro-Mendoza CK, Tomashek KM, Kotelchuck M, et al. Effect of late-preterm birth and maternal medical conditions on newborn morbidity risk. Pediatrics 2008;121:e223-232. [PubMed: 18245397]

13. Tomashek KM, Shapiro-Mendoza CK, Davidoff MJ, et al. Differences in mortality between latepreterm and term singleton infants in the United States, 1995-2002. J Pediatr 2007;151:450-456. e451. [PubMed: 17961684]

14. Wang ML, Dorer DJ, Fleming MP, et al. Clinical outcomes of near-term infants. Pediatrics 2004;114:372-376. [PubMed: 15286219]

15. Raju TN, Higgins RD, Stark AR, et al. Optimizing care and outcome for late-preterm (near-term) infants: a summary of the workshop sponsored by the National Institute of Child Health and Human Development. Pediatrics 2006;118:1207-1214. [PubMed: 16951017]

16. Zaidi AK, Harrell LJ, Rost JR, et al. Assessment of similarity among coagulase-negative staphylococci from sequential blood cultures of neonates and children by pulsed-field gel electrophoresis. J Infect Dis 1996;174:1010-1014. [PubMed: 8896502]

17. Stoll BJ, Hansen N, Fanaroff AA, et al. Changes in pathogens causing early-onset sepsis in very-lowbirth-weight infants. N Engl J Med 2002;347:240-247. [PubMed: 12140299]

18. Stoll BJ, Gordon T, Korones SB, et al. Early-onset sepsis in very low birth weight neonates: a report from the National Institute of Child Health and Human Development Neonatal Research Network. J Pediatr 1996;129:72-80. [PubMed: 8757565]

19. Diminishing racial disparities in early-onset neonatal group B streptococcal disease--United States, 2000-2003. MMWR Morb Mortal Wkly Rep 2004;53:502-505. [PubMed: 15201842]

20. Schrag SJ, Zywicki S, Farley MM, et al. Group B streptococcal disease in the era of intrapartum antibiotic prophylaxis. N Engl J Med 2000;342:15-20. [PubMed: 10620644]

21. Ronnestad A, Abrahamsen TG, Medbo S, et al. Septicemia in the first week of life in a Norwegian national cohort of extremely premature infants. Pediatrics 2005;115:e262-268. [PubMed: 15687417]

22. Benjamin DK, DeLong E, Cotten CM, et al. Mortality following blood culture in premature infants: increased with Gram-negative bacteremia and candidemia, but not Gram-positive bacteremia. J Perinatol 2004;24:175-180. [PubMed: 14985775]

23. Bizzarro MJ, Raskind C, Baltimore RS, et al. Seventy-five years of neonatal sepsis at Yale: 1928-2003. Pediatrics 2005;116:595-602. [PubMed: 16140698]

24. Jiang JH, Chiu NC, Huang FY, et al. Neonatal sepsis in the neonatal intensive care unit: characteristics of early versus late onset. J Microbiol Immunol Infect 2004;37:301-306. [PubMed: 15497012]

25. Aziz K, McMillan DD, Andrews W, et al. Variations in rates of nosocomial infection among Canadian neonatal intensive care units may be practice-related. BMC Pediatr 2005;5:22. [PubMed: 16004613]

26. Cordero L, Sananes M, Ayers LW. Bloodstream infections in a neonatal intensive-care unit: 12 years' experience with an antibiotic control program. Infect Control Hosp Epidemiol 1999;20:242-246. [PubMed: 10219874]

27. Benjamin DK Jr, Stoll BJ, Fanaroff AA, et al. Neonatal candidiasis among extremely low birth weight infants: risk factors, mortality rates, and neurodevelopmental outcomes at 18 to 22 months. Pediatrics 2006;117:84-92. [PubMed: 16396864] 
28. Gordon A, Isaacs D. Late onset neonatal Gram-negative bacillary infection in Australia and New Zealand: 1992-2002. Pediatr Infect Dis J 2006;25:25-29. [PubMed: 16395098]

29. Karlowicz MG, Buescher ES, Surka AE. Fulminant late-onset sepsis in a neonatal intensive care unit, 1988-1997, and the impact of avoiding empiric vancomycin therapy. Pediatrics 2000;106:13871390. [PubMed: 11099593] 


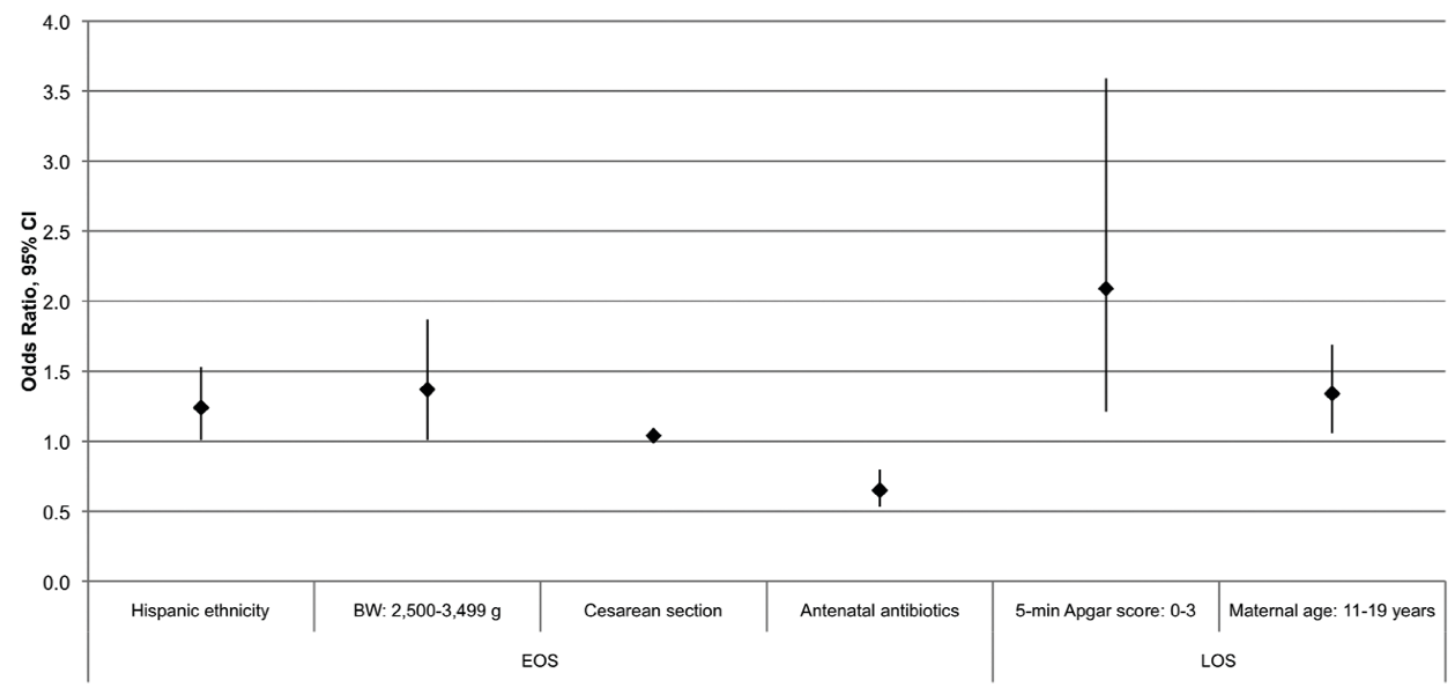

Figure 1.

Odds ratios for early and late onset sepsis associated with selected variables among late preterm infants by multivariable regression analysis. BW indicates birth weight; $\mathrm{CI}$, confidence interval; EOS, early onset sepsis; LOS, late onset sepsis. 


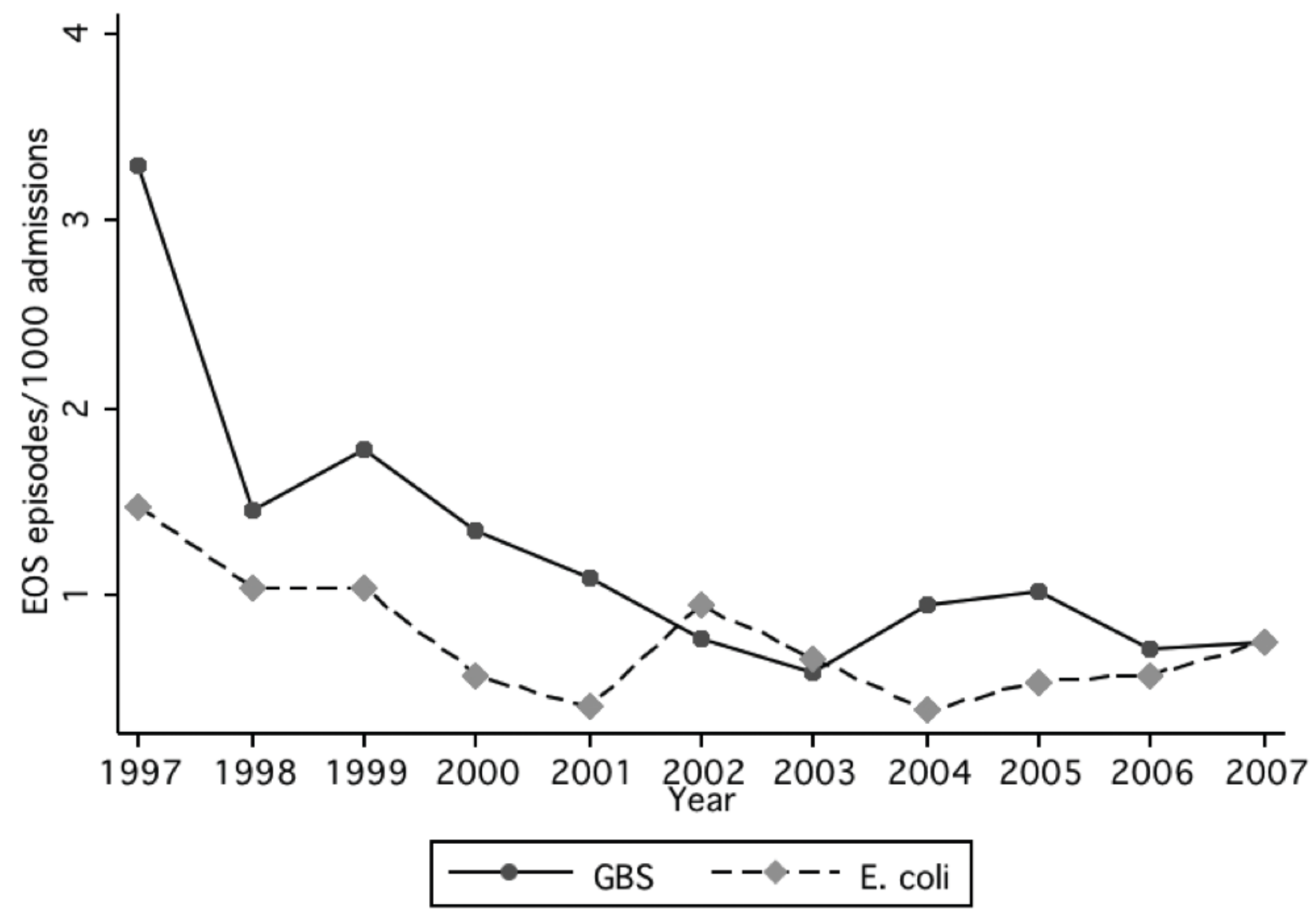

Figure 2.

Group-B streptococci and Escherichia coli early onset sepsis annual cumulative incidence per 1000 late preterm NICU admissions between 1997 and 2007. EOS indicates early onset sepsis; GBS, group-B streptococci. 


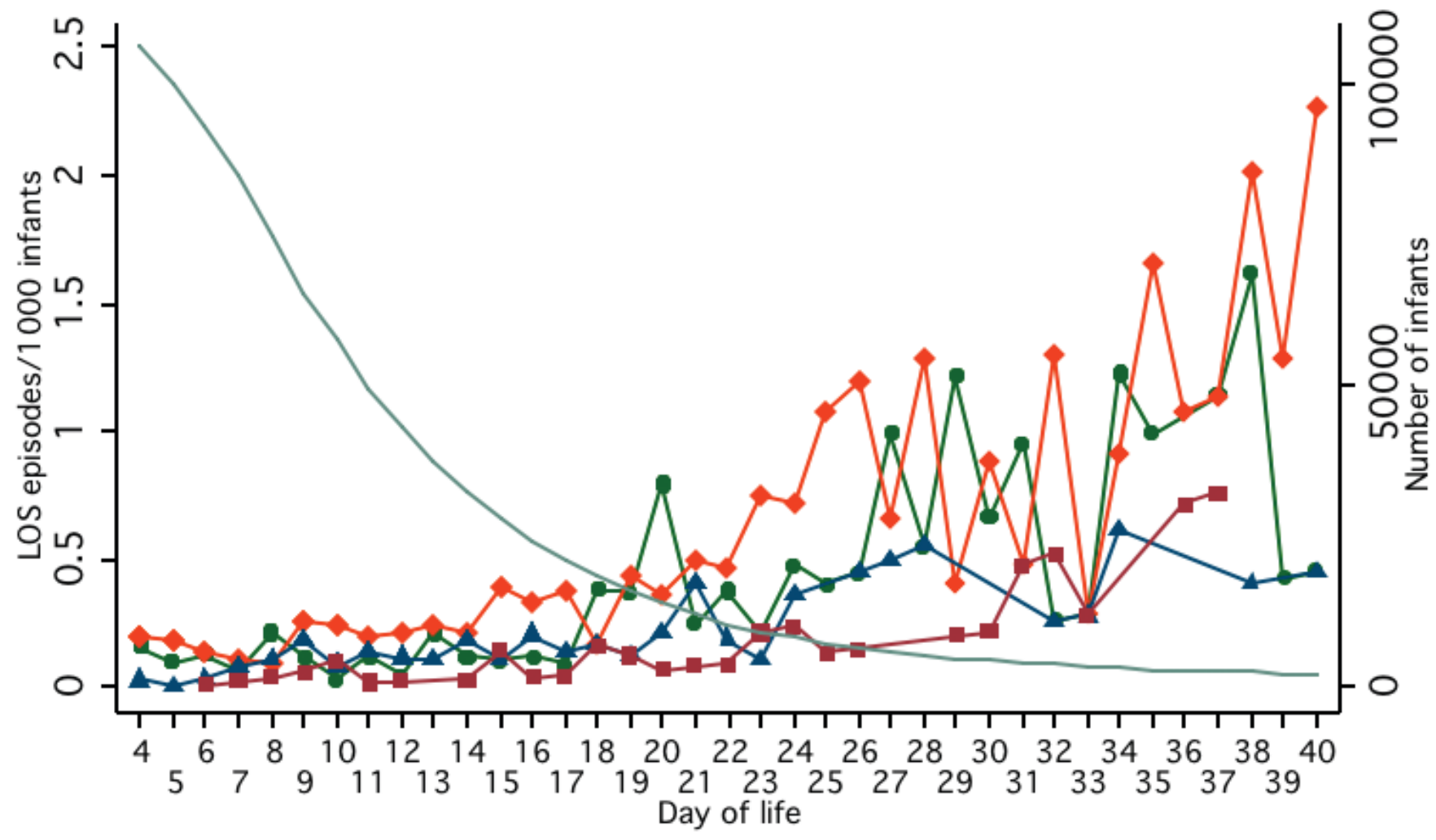

Day of life

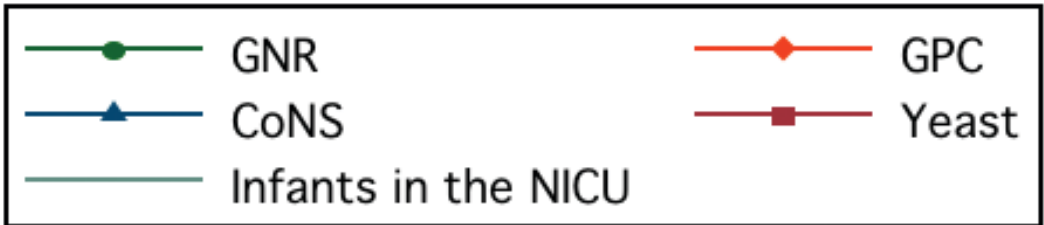

Figure 3.

Infection episodes by organism group and postnatal age among late preterm infants with late onset sepsis. CoNS indicates coagulase-negative Staphylococcus; GNR, Gram-negative rods; GPC, Gram-positive cocci; LOS, late-onset sepsis; NICU, neonatal intensive care unit. 
Table 1

Demographics of Late Preterm Infants with Early and Late Onset Sepsis

\begin{tabular}{|c|c|c|}
\hline Characteristic & $\begin{array}{c}\text { Infants with } \operatorname{EOS}(\mathrm{N}= \\
\mathbf{5 2 7})\end{array}$ & $\begin{array}{c}\text { Infants with } \operatorname{LOS}(\mathrm{N}= \\
669)\end{array}$ \\
\hline \multicolumn{3}{|l|}{ Race/ethnicity, n (\%) } \\
\hline White & $274(55)$ & $310(46)$ \\
\hline Black & $62(12)$ & $72(11)$ \\
\hline Hispanic & $147(28)$ & $214(32)$ \\
\hline Other ${ }^{*}$ & $15(3)$ & $41(6)$ \\
\hline Unknown & $29(6)$ & $32(5)$ \\
\hline \multicolumn{3}{|l|}{ Sex, n (\%) } \\
\hline Female & $230(44)$ & $299(45)$ \\
\hline Male & $297(56)$ & $367(55)$ \\
\hline \multicolumn{3}{|l|}{ Gestational age (weeks), n (\%) } \\
\hline 34 & $178(34)$ & $256(38)$ \\
\hline 35 & $152(29)$ & $212(32)$ \\
\hline 36 & 197 (37) & $201(30)$ \\
\hline \multicolumn{3}{|l|}{ Birth weight (grams), n (\%) } \\
\hline$<2000$ & $65(12)$ & $179(27)$ \\
\hline $2000-2499$ & $210(40)$ & $256(38)$ \\
\hline $2500-3499$ & $230(44)$ & $201(30)$ \\
\hline$>3499$ & $21(4)$ & $29(4)$ \\
\hline Unknown & - & $4(1)$ \\
\hline \multicolumn{3}{|l|}{ Apgar score at $5 \min , \mathrm{n}(\%)$} \\
\hline $0-3$ & $6(1)$ & $17(3)$ \\
\hline $4-6$ & $33(6)$ & $50(7)$ \\
\hline $7-10$ & $478(91)$ & $578(86)$ \\
\hline Unknown & $10(2)$ & $24(4)$ \\
\hline Inborn, n (\%) & $408(77)$ & $471(70)$ \\
\hline \multicolumn{3}{|l|}{ Maternal age (years), n (\%) } \\
\hline $11-19$ & $75(14)$ & $119(18)$ \\
\hline $20-29$ & $273(52)$ & $328(49)$ \\
\hline $30-39$ & $156(30)$ & $194(29)$ \\
\hline$>39$ & $20(4)$ & $22(3)$ \\
\hline Unknown & $3(1)$ & $6(1)$ \\
\hline \multicolumn{3}{|l|}{ Delivery, n (\%) } \\
\hline Vaginal & $265(50)$ & $276(41)$ \\
\hline Cesarean section & $252(48)$ & $386(58)$ \\
\hline Unknown & $10(2)$ & $7(1)$ \\
\hline Antenatal steroids received, $\mathrm{n}(\%)$ & $87(17)$ & $130(19)$ \\
\hline Antenatal antibiotics received, n (\%) & $154(29)$ & $190(28)$ \\
\hline
\end{tabular}

EOS indicates early onset sepsis; LOS indicates late onset sepsis.

* Other race/ethnicity includes American/Alaskan Native, Asians, and other

Pediatr Infect Dis J. Author manuscript; available in PMC 2010 December 1. 


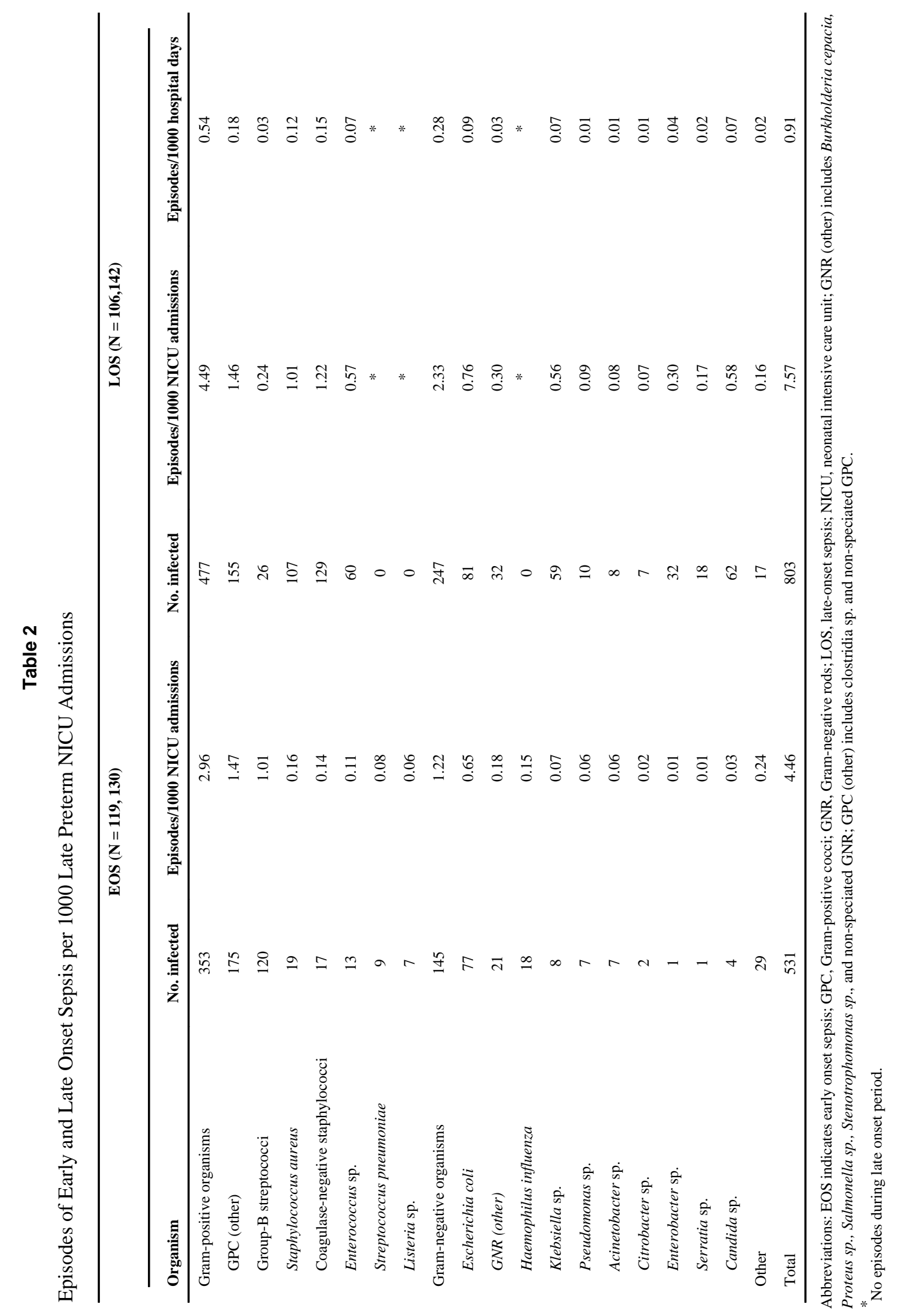

Pediatr Infect Dis J. Author manuscript; available in PMC 2010 December 1. 